\title{
Pictures from the Endeavour voyage of 1768
}

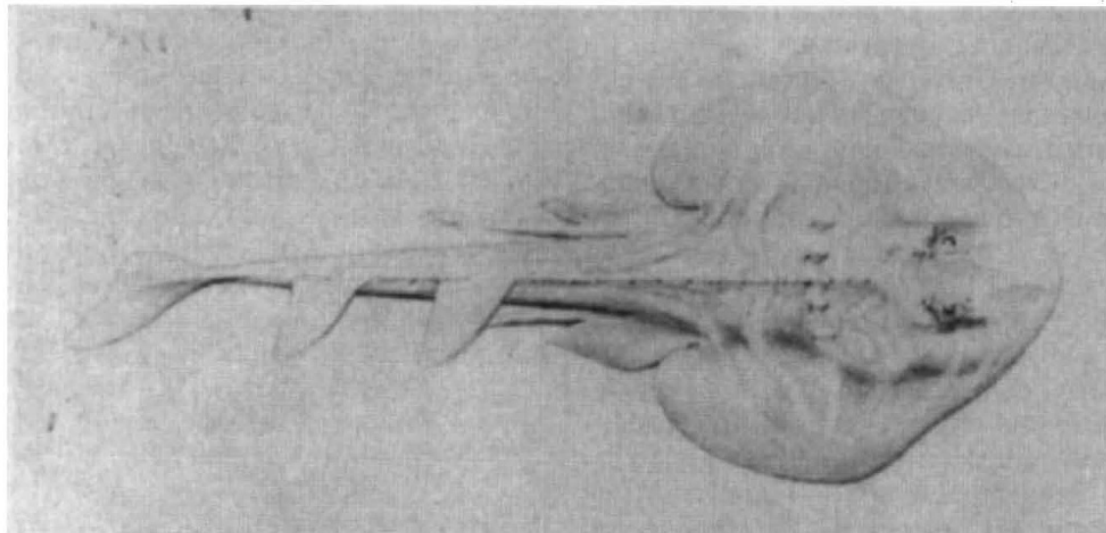

Trygonorhina fasciata Müller and Henle, 1841. Drawing by Spöring of a fish caught on 29 April 1770 at Sting Ray Bay (later Botany Bay), Australia. T. fasciata was based by Müller and Henle partly on this drawing, but also on another specimen in alcohol at the Paris museum. From Catalogue of the Natural History Drawings commissioned by Joseph Banks on the Endeavour Voyage 1768-1771 Part 3: Zoology, recently published by the Bulletin of the British Museum (Natural History). (Historical series vol. 13; London, 1986.) This catalogue lists all the drawings of animals from James Cook's voyage on the Endeavour that were made by artists employed by Joseph Banks. Most of the drawings were kept in Banks' home until his death in 1820 and then transferred to the Museum. This catalogue attempts to record comprehensively the surviving animal drawings from the voyage of the Endeavour, and examines and discusses the history of the drawings as well as providing notes on the artists, none of whom survived the voyage.

least some of these minimal antigens interact physically with class II molecules in the absence of the T-cell receptor, providing an understandable picture of how simultaneous recognition of the two is achieved.

This view of antigen presentation to class II-restricted T cells diverges markedly from the present view of antigen recognition by class I-restricted T lymphocytes. especially cytotoxic T lymphocytes (CTL). These cells seem specific for integral cell-membrane molecules such as viral envelope glycoproteins, allogeneic MHC molecules, or minor histocompatibility antigens that have been assumed (without any experimental evidence) to be uncharacterized membrane proteins. Immunologists presumed that these intact membrane proteins interacted directly with class I MHC molecules on the cell surface. However, such a marked difference between class I and class II antigen interaction became less and less likely as accumulating data showed striking similarities between the relationship of both class I and class II molecular structures to T-cell recognition ${ }^{13}$, and the use of the same $V$ and $V_{1}$ gene segments by the receptors of class I- and class II-restricted T cells"

\section{Experiments}

The experiments of Townsend et al. now reveal that these differences are more imagined than real, and that similar (though not necessarily identical) forms of antigen participate in recognition by all MHC-restricted $T$ cells. The new findings come from work on the specificity of both human and murine CTL generated by immunization with the influenza virus. Early studies ${ }^{2}$ demonstrated many CTL clones that recognized specificities shared by distinct serotypes of this virus. The viral protein accounting for this crossreactivity was, unexpectedly, the nucleoprotein and not the membrane-expressed haemagglutinin molecule. Confirmation of these results was obtained using L cells co-transfected with the proper class I gene and the nucleoprotein gene. These cells were recognized appropriately by the cross-reactive CTL clones'.

Given that interaction of CTL receptors with target cells occurs at the outer surface of the cell membrane, how can a molecule that does not appear on the membrane serve as a target antigen? This conundrum was partially solved by the demonstration that transfectants expressing truncated forms of the nucleoprotein molecule could serve as adequate CTL targets ${ }^{4}$. Finally, the experiments described in their most recent paper ${ }^{7}$ show that, just as synthetic peptides could be used to stimulate class II-restricted T cells, target cells exposed in vitro to short synthetic peptides corresponding to certain regions of the nucleoprotein molecule could be recognized by nucleoprotein-specific CTL. The active peptides, as would be expected, correspond to regions of nucleoprotein sequence of the immunizing strain of virus that differ from the sequence of nucleoprotein of a strain of virus incapable of sensitizing cells for recognition by these particular CTL clones. Thus, it appeared unnecessary for the intact nucleoprotein mysteriously to reach the cell surface. Instead, some fragmented or degraded form probably serves as the antigen after travelling to the membrane by a mechanism independent of the normal intracellular sorting process acting on the intact nucleoprotein.

\section{Similarities}

Thus, we now have a satisfying similarity in the general nature of antigen recognized by both class I- and class IIrestricted $\mathrm{T}$ cells. The new data on CTL recognition also provide an explanation for the repeated failure of investigators to generate antibodies to minor histocompatibility antigens. Rather than constituting a family of intrinsic cell-membrane molecules, these antigens are more likely to represent processed fragments of cytoplasmic or nuclear proteins that normally never reach the membrane in an intact state, just as with influenza nucleoprotein. Even if antibodies were raised in response to the very small amount of such fragments that probably are present on the cell surface, they are not likely to be detected in binding or cytotoxcity assays.

The studies on influenza-specific CTL support the emerging view that antigen processing is not a specialized function of a small subset of class II-bearing, bone marrow-derived cells, but rather the reflection of a more fundamental set of intracellular activitites occurring in virtually all cells. An early hint that this might be the case came from experiments showing that fibroblasts made to express class II antigens by DNA-mediated gene transfer could also present various protein antigens to T cells, and that this function is susceptible to the same inhibitors that interfere with processing by haematopoietic-presenting cells ${ }^{13}$. The demonstration by Townsend et al. ${ }^{5}$ that class Irestricted $\mathrm{T}$ cells are specific for antigen fragments suggests that most, if not all, cells have some capacity for antigen processing, because class I molecules are widely distributed on various somatic cell types, and CTL recognize virally infected or minor histocompatibility antigenbearing cells of diverse tissue origin.

Is the same intracellular pathway used for processing those antigens taken up from the external environment of the cell and those synthesized endogenously? Do both classes of $\mathrm{MHC}$ molecules interact with or present the same processed antigens? Recently published work by Morrison et al. ${ }^{14}$ suggests the answer to both of these questions may be 'no'. These investigators find that influenza haemagglutinin sythesized endogenously can not be recognized by class II-restricted, haemagglutinin-specific $\mathrm{T}$-cell clones, but provide an adequate target for class I-restricted cells. Conversely, the class I-restricted 